\title{
Bouncing Cosmologies and Self-Gravitational Corrections
}

\author{
M.R. Setare * \\ Physics Dept. Inst. for Studies in Theo. Physics and Mathematics(IPM) \\ P. O. Box 19395-5531, Tehran, IRAN
}

\begin{abstract}
A four-dimensional timelike brane is considered as the boundary of the $S A d S_{5}$ bulk background. Exploiting the CFT/FRW-cosmology relation, we derive the selfgravitational corrections to the first Friedmann-like equation which is the equation of the brane motion. The additional term that arises due to the semiclassical analysis, can be viewed as stiff matter where the self-gravitational corrections act as the source for it. This result is contrary to standard analysis that regards the charge of $S A d S_{5}$ bulk black hole as the source for stiff matter. A very interesting feature of the solutions of FRW equation is that they all (for $k=0, \pm 1$ ) have a non-vanishing minimum value for the scale factor.
\end{abstract}

\footnotetext{
*E-mail: rezakord@ipm.ir
} 


\section{Introduction}

Black hole thermodynamic quantities depend on the Hawking temperature via the usual thermodynamic relations. The Hawking temperature undergoes corrections from many sources:the quantum corrections, the self-gravitational corrections, and the corrections due to the generalized uncertainty principle.

There has been much recent interest in calculating the quantum corrections to $S_{B H}$ (the Bekenestein-Hawking entropy) [1]-18. The leading-order correction is proportional to $\ln S_{B H}$. There are, two distinct and separable sources for this logarithmic correction [12, 13] (see also recent paper by Gour and Medved [15]). Firstly, there should be a correction to the number of microstates that is a quantum correction to the microcanonical entropy, secondly, as any black hole will typically exchange heat or matter with its surrounding, there should also be a correction due to thermal fluctuations in the horizon area. In a recent work Carlip [2] has deduced the leading order quantum correction to the classical Cardy formula. The Cardy formula follows from a saddle-point approximation of the partition function for a two-dimensional conformal field theory. This leads to the theory's density of states, which is related to the partition function by way of a Fourier transform [19].

Concerning the quantum process called Hawking effect [20] much work has been done using a fixed background during the emission process. The idea of Keski-Vakkuri, Kraus and Wilczek (KKW) [21] is to view the black hole background as dynamical by treating the Hawking radiation as a tunnelling process. The energy conservation is the key to this description. The total (ADM) mass is kept fixed while the mass of the black hole under consideration decreases due to the emitted radiation. The effect of this modification gives rise to additional terms in the formulae concerning the known results for black holes [22]; a nonthermal partner to the thermal spectrum of the Hawking radiation shows up.

The generalized uncertainty principle corrections are not tied down to any specific model of quantum gravity; these corrections can be derived using arguments from string theory [23] as well as other approaches to quantum gravity [24].

In recent years, there has been an enormous amount of research into two important areas of the theoretical physics:branworld cosmology and the holographic principle. The braneworld scenario gained momentum as a solution to the hierarchy problem [25, 26, 27. The holographic principle, meanwhile, was first realized in string theory via the AdS/CFT correspondence 28, 29, 30. Much attention has currently been paid for the duality between de Sitter (dS) gravity and CFT in analogy to the AdS/CFT correspondence [31](for a very good review see also [32]). The essence of braneworld holography [33]-37] can be captured in the following claim : Randall-Sundrum branworld gravity is dual to a CFT with a UV cutoff, coupled to gravity on the brane. Formal evidence for this claim was provided by studying a brane universe in the background of the Schwarzschild-AdS Bblack hole. The introduction of the black hole on the gravity side of the AdS/CFT correspondence corresponds to considering finite temperature states in the dual CFT [38. In the context of braneworld holography, Savonije and Verlinde demonstrated that their induced braneworld cosmology could alternatively be described as the standard FRW cosmology driven by the energy density of this dual CFT [39, 34]

In the present paper, we take into account corrections to the entropy of the fivedimensional Schwarzschild- anti de Sitter black hole (abbreviated to $S A d S_{5}$ in the sequel) 
that arise due to the self-gravitational effect. Previous studies of the Cardy-Verlinde formula (or the corresponding Friedmann equation) in an AdS/CFT context have attracted a lot of attention. For instance, the author with Vagenas have shown that the entropy of Achúcarro-Ortiz black hole (a locally AdS space) can be described by the Cardy-Verlinde formula [40] and the self-gravitational corrections to this formula have been computed [41. By introducing the self-gravitational corrections to the Cardy-Verlinde formula, we find a host of interesting cosmological solutions for the brane universe. Their most remarkable feature is that the self-gravitational corrections allows for a nonsingular transition between a contracting phase of the scale factor of the brane, and a following expanding stage.

\section{Self-Gravitational Corrections to Cardy-Verlind For- mula}

In the asymptotic coordinates, the $S A d S_{5}$ black hole metric is

$$
d s^{2}=-F(r) d t^{2}+\frac{1}{F(r)} d r^{2}+r^{2} d \Omega_{(3)}^{2},
$$

where

$$
F(r)=1-\frac{\mu}{r^{2}}+r^{2}
$$

and we work in units where the $A d S$ radius $l=1$. The parameter $\mu$ is proportional to the ADM mass $M$ of the black hole. The free energy, $F$, the entropy, $S$, and the thermodynamical energy $E$, of the black hole are given respectively,

$$
F=\frac{-V_{3} r_{H}^{2}}{16 \pi G_{5}}\left(r_{H}^{2}-1\right), \quad S=\frac{V_{3} r_{H}^{3}}{4 G_{5}}, \quad E=F+T_{H} S=\frac{3 V_{3} \mu}{16 \pi G_{5}}
$$

where $G_{5}$ is Newton's constant in 5 dimensions and $V_{3}$ is the volume of a unit 3 -sphere, and $r_{H}$ is the horizon radius.

We are interested primarily in the corrections to the entropy (3) that arise in the context of KKW analysis [21 ${ }^{1}$ due to self-gravitational effect. We take into account the response of the background geometry to an emitted quantum of frequency $\omega$. The particle travels across the horizon from $r_{i}$ to $r_{f}$ which are given by

$$
\begin{gathered}
r_{i}=\sqrt{1 / 2(\sqrt{1+4 \mu}-1)} \\
r_{f}=\sqrt{1 / 2\left(\sqrt{1+4 \mu^{\prime}}-1\right)} .
\end{gathered}
$$

To first order in the emitted energy $(\omega)$, the afore-mentioned radii are related as follows

$$
r_{f}^{2}=r_{i}^{2}\left(1-\omega \frac{16 \pi G_{5}}{3 V_{3} r_{i}^{2} \sqrt{1+4 \mu}}\right)
$$

\footnotetext{
${ }^{1}$ Further developments in KKW analysis can be found in [22].
} 
Consequently, the change in the entropy of the $S A d S_{5}$ black hole during the process of emission takes the form

$$
\Delta S=S_{i}-S_{f}=\frac{V_{3}}{4 G_{5}}\left(r_{i}^{3}-r_{f}^{3}\right)=\frac{\omega}{T(\omega)}
$$

where $S_{f}$ is the modified entropy of the $S A d S_{5}$ black hole due to the self-gravitational effect, $S_{i}$ is the standard formula for the entropy (Bekenstein-Hawking entropy) derived when the black hole mass is kept fixed, and $T(\omega)$ is the corrected temperature of the black hole horizon $T_{H}$ due to the self-gravitational effect. The expression for the modified temperature of the black hole horizon is given as

$$
T(\omega)=\frac{\left(2 r_{i}^{2}+1\right)}{2 \pi r_{i}}+\mathcal{O}(\omega)
$$

To first order in $\omega$, the corrected entropy of the $S A d S_{5}$ black hole reads

$$
S_{f}=S_{i}-\frac{2 \pi r_{i}}{\left(2 r_{i}^{2}+l^{2}\right)} \omega
$$

Due to the AdS/CFT correspondence, we are now ready to evaluate the corrections to the Cardy-Verlinde formula for the entropy of the $S A d S_{5}$ black hole. The Casimir energy for the spacetime under study is given by

$$
E_{C}=4 E_{4}-3 T_{H} S_{i}
$$

where the four-dimensional energy $E_{4}$, is given by 39]

$$
E_{4}= \pm \frac{3 V_{3}}{16 \pi G_{5} r} \mu=\frac{1}{r} E
$$

where + corresponds to the black hole horizon and - to the cosmological horizon. It is easily seen that, to first order in $\omega$, the modified Casimir energy takes the form

$$
\mathcal{E}_{C}=E_{C}-\frac{1}{r} \omega
$$

Due to the self-gravitational corrections, the modified Cardy-Verlinde formula for the entropy of the $S A d S_{5}$ black hole is given as

$$
\mathcal{S}_{C F T}=\frac{2 \pi r}{3} \sqrt{\left|\mathcal{E}_{C}\left(2 \mathcal{E}_{4}-\mathcal{E}_{C}\right)\right|}
$$

Using equations (10) and (12), the modified Cardy-Verlinde entropy formula becomes

$$
\mathcal{S}_{C F T}=\frac{2 \pi r}{3} \sqrt{\left|\left[E_{C}-\frac{1}{r} \omega\right]\left[\left(2 E_{4}-E_{C}\right)-\frac{1}{r} \omega\right]\right|}
$$

and keeping terms up to first order in the emitted energy $\omega$, it takes the form

$$
\mathcal{S}_{C F T}=S_{C F T}(1-\varepsilon \omega)
$$


where the small parameter $\varepsilon$ is given by

$$
\varepsilon=\frac{1}{r} \frac{E_{4}}{E_{C}\left(2 E_{4}-E_{C}\right)} .
$$

A welcomed but not unexpected result is that there is no entropy bound violation due to self-gravitational corrections to the Cardy-Verlinde entropy ${ }^{2}$.

\section{Self-Gravitational Corrections to FRW Brane Cos- mology}

We now consider a 4-dimensional brane in the $S A d S_{5}$ black hole background. This 4dimensional brane can be regarded as the boundary of the 5-dimensional $S A d S_{5}$ bulk background. Let us first replace the radial coordinate $r$ with $a$ and so the line element (11)

$$
d s^{2}=-F(a) d t^{2}+\frac{1}{F(a)} d a^{2}+a^{2} d \Omega_{(3)}^{2},
$$

It was shown that by reduction from the $S A d S_{5}$ background (17) and by imposing the condition

$$
-F(a)\left(\frac{\partial t}{\partial \tau}\right)^{2}+\frac{1}{F(a)}\left(\frac{\partial a}{\partial \tau}\right)^{2}=-1
$$

where $\tau$ is a new time parameter, one obtains an FRW metric for the 4-dimensional timelike brane

$$
d s_{(4)}^{2}=-d \tau^{2}+a^{2}(\tau) d \Omega_{(3)}^{2} .
$$

Thus, the 4-dimensional FRW equation describes the motion of the brane universe in the $S A d S_{5}$ background. It is easy to see that the matter on the brane can be regarded as radiation and consequently, the field theory on the brane should be a CFT.

Within the context of context the AdS/CFT correspondence, Savonije and Verlinde studied the CFT/FRW-cosmology relation from the Randall-Sundrum type braneworld perspective [34. They showed that the entropy formulas of the CFT coincides with the Friedmann equations when the brane crosses the black hole horizon.

In the case of a 4-dimensional timelike

$$
d s_{(4)}^{2}=-d \tau^{2}+a^{2}(\tau) d \Omega_{(3)}^{2},
$$

one of the identifications that supports the CFT/FRW-cosmology relation is

$$
H^{2}=\left(\frac{2 G_{4}}{V}\right)^{2} \mathcal{S}^{2}
$$

where $H$ is the Hubble parameter defined by $H=\frac{1}{a} \frac{d a}{d \tau}$ and $\mathrm{V}$ is the volume of the 3 -sphere $\left(V=a^{3} V_{3}\right)$. The 4-dimensional Newton constant $G_{4}$ is related to the 5-dimensional one $G_{5}$ by

$$
G_{4}=\frac{2}{l} G_{5} .
$$

\footnotetext{
${ }^{2} \mathrm{~A}$ violation of the holographic entropy bound was observed when self-gravitational corrections to the Cardy-Verlinde entropy formula of the two-dimensional Achúcarro-Ortiz black hole were included [41] (see also [45]).
} 
It was shown that at the moment that the 4-dimensional timelike brane crosses the cosmological horizon, i.e. when $a=a_{b}$, the CFT entropy and the entropy of the $S A d S_{5}$ black hole are identical. By substituting (15) into (21), we obtain the self-gravitational corrections to the motion of the CFT-dominated brane

$$
H^{2}=\left(\frac{2 G_{4}}{V}\right)^{2} S_{C F T}^{2}(1-\varepsilon \omega)^{2} .
$$

It is obvious that from the first term on the right-hand side of (23) we get the standard Friedmann equation with the appropriate normalization

$$
H^{2}=\frac{-1}{a_{b}^{2}}+\frac{8 \pi G_{4}}{3} \rho
$$

where $\rho$ is the energy density defined by $\rho=E_{4} / V$. Therefore, the correction to the FRW equation due to the self-gravitation effect is expressed by the second term in the right-hand side of equation (23). Keeping terms up to first order in the emitted energy $(\omega)$, the modified Hubble equation due to the self-gravitation corrections is

$$
H^{2}=\frac{-1}{a_{b}^{2}}+\frac{8 \pi G_{4}}{3} \rho-2 E_{4} \frac{1}{a_{b}}\left(\frac{2 G_{4}}{V}\right)^{2}\left(\frac{2 \pi a_{b}}{3}\right)^{2} \omega .
$$

Taking into account that all quantities should be evaluated on the black hole horizon, the modified Hubble equation, i.e. the first Friedmann equation, takes the form

$$
H^{2}=\frac{-1}{a_{b}^{2}}+\frac{8 \pi G_{4}}{3} \rho-\frac{8 \pi G_{4}}{3}\left[\frac{4 \pi G_{4}}{3} \frac{1}{a_{b}^{2} V_{3}} \rho\right] \omega
$$

where the volume $V$ is given by $a_{b}^{3} V_{3}$. At this point it should be stresses that our analysis was up to now restricted to the spatially flat $(k=+1)$ spacelike brane.

We will now extend the aforesaid analysis. We therefore consider an arbitrary scale factor $a$ and include a general $k$ taking values $+1,0,-1$ in order to describe, respectively, the elliptic, flat, and hyperbolic horizon geometry of the $S A d S_{5}$ bulk black hole. The modified Hubble equation is now given by

$$
H^{2}=\frac{-k}{a^{2}}+\frac{8 \pi G_{4}}{3} \rho-\frac{8 \pi G_{4}}{3}\left[\frac{4 \pi G_{4}}{3} \frac{1}{a^{2} V_{3}} \rho\right] \omega
$$

where the volume $V$ is now given by $a^{3} V_{3}$ since all quantities that appear in equation (27) are defined for an arbitrary scale factor $a$.

The first term in the right-hand side of equation (27) represents the curvature contribution to the brane motion. The second term can be regarded as the contribution from the radiation and it redshifts as $a^{-4}$. The last term in the right-hand side of equation (27) is the self-gravitational correction to the motion of 4-dimensional timelike brane moving in the 5-dimensional Schwarzschild-anti de Sitter bulk background. Since this term goes like $a^{-6}$, it is obvious that it is dominant at early times of the brane evolution while at late times the second term, i.e. the radiative matter term, dominates and thus the last term can be neglected. The sign of last term is opposite with respect to the standard situation, one may expect that this sign difference could have interesting cosmological consequences. The bounce can be attributed to the negative-energy matter, which dominates at small values of $a$ and create a significant enough repulsive force so that a big 
crunch is avoided. Indeed, we will see that it is crucial in allowing a nonsingular transition between a contracting and an expanding evolution of the scale factor $a$.

We would like to regard the last term in right-hand side of equation (27) as stiff matter [49. In particular, within the context of AdS/CFT correspondence,Mukherji, Peloso [46] and Medved 47] have considered a Reissner-Nordstrom-anti de Sitter bulk background 50

$$
d s^{2}=-h(a) d t^{2}+\frac{1}{h(a)} d a^{2}+a^{2} d \Omega_{(3)}^{2}
$$

where

$$
h(a)=k+\frac{a^{2}}{l^{2}}-\frac{\varepsilon_{3} M}{a^{2}}+\frac{3 \varepsilon_{3}^{2} Q^{2}}{16 a^{4}} .
$$

where

$$
\varepsilon_{3}=\frac{16 \pi G_{5}}{3 V_{3}}
$$

and $M, Q$ represent the black hole mass and charge, respectively.

For charged black hole bulk, the brane world turns out to be a bounce cosmology 48, 46. 47. That is, the universe is asymptotically de Sitter in the far past, contracts to a non-vanishing minimum at some given time and then expands to an asymptotically de Sitter future. It was showed that the brane evolution is described by a Friedmann-like equation for radiative matter along with a stiff-matter contribution

$$
\begin{aligned}
H^{2} & =\frac{-k}{a^{2}}+\frac{\varepsilon_{3} M}{a^{4}}-\frac{3 \varepsilon_{3}^{2} Q^{2}}{16 a^{6}} \\
& =\frac{-k}{a^{2}}+\frac{8 \pi G_{4}}{3} \rho-\frac{3 \varepsilon_{3}^{2} Q^{2}}{16 a^{6}} .
\end{aligned}
$$

If the following condition is satisfied

$$
Q^{2}=\frac{8}{3} M \omega
$$

then equations (27) and (31) are identical and the last term in right-hand side of equation (27) is then regarded as stiff matter.

The evolution of the system can be solved exactly, as one can most simply realize by using conformal time $\eta$, defined as $d t=a(\eta) d \eta$. Let us first consider a closed four dimensional geometry. In this case the solution is

$$
a(\eta)=\sqrt{\frac{\varepsilon_{3} M}{2}}\left[1-C_{1} \cos (2 \eta)\right]^{1 / 2}, \quad C_{1}=\sqrt{1-\frac{2 \omega}{M}} .
$$

$C_{1}$ is real because $2 \omega \ll M$. Hence, the universe evolves periodically, with a four dimensional radius oscillating between a maximal and a minimal size given by

$$
a_{\max , \min }=\sqrt{\frac{\varepsilon_{3} M}{2}}\left(1 \pm C_{1}\right)^{1 / 2} .
$$

Notice that we have used the freedom in setting the origin of conformal time so that the minimal radius is reached at $\eta=n \pi$, where $n$ is an integer number. From the bulk perspective, the brane starts out from the black hole at a distance $a_{\min }$ from the singularity 
and moves away up to $a_{\max }$ as it expands. At later time, it collapses at $a=a_{\min }$. It is interesting to compare the case at hand and the situation without self-gravitational corrections. In the latter situation, the cosmological evolution is governed by Eq.(27), but with $\omega=0$. This, in turn, means that the time dependence of the scale factor is given by Eq.(33), with $C_{1}=1$. In this case, the scale factor starts from zero size and expands up to $a_{\max }$ before collapsing again to zero size. From the bulk point of view, the brane originates from the black hole singularity and at a latter stage it collapses again into the singularity. We, therefore, see that the effect of self-gravitational corrections is rather non-trivial, since it make the cosmology of the model free from singularities.

For an open universe, we find the solution

$$
a(\eta)=\sqrt{\frac{\varepsilon_{3} M}{2}}\left[C_{2} \cosh (2 \eta)-1\right]^{1 / 2}, \quad C_{2}=\sqrt{1+\frac{2 \omega}{M}} .
$$

In this case, the brane is initially contracting, and then bounces to an expanding phase. Again, we have set $\eta=0$ at the bounce. The minimal radius is given by

$$
a_{m i n}=\sqrt{\frac{\varepsilon_{3} M}{2}}\left(C_{2}-1\right)^{1 / 2}
$$

As before, $a_{\min } \rightarrow 0$ as $\omega \rightarrow 0$.

Finally, in the case of a flat universe we find

$$
a(\eta)=\sqrt{\varepsilon_{3}\left(M \eta^{2}+\frac{\omega}{2}\right)}
$$

Also in this case we have a bouncing universe, with a minimal radius

$$
a_{\min }=\sqrt{\frac{\varepsilon_{3}}{2} \omega}
$$

At late times one recovers the evolution $a(\eta) \sim \eta \sim t^{1 / 2}$, which is typical of a flat universe dominated by radiation.

It is clear from the above forms that, as long as the self-gravitational corrections are non-vanishing, $a$ will never shrink to zero. At the bounce $(t=\eta=0)$ in particular, all three equations take the simple form

$$
a(0)=\sqrt{\frac{\varepsilon_{3}}{2} \omega}+O\left(\omega^{2}\right) .
$$

Therefore, the feature of a bouncing universe would allow one to circumvent the issue of resolving the big bang (or crunch) singularity, which afflicts many (if not most) cosmological models.

\section{Conclusions}

One of the striking results for the dynamic AdS/CFT correspondence is that the CardyVerlinde's formula on the CFT-side coincides with the first Friedmann equation (Hubble equation) in cosmology when the brane crosses the horizon $a=a_{b}$ of the $S A d S_{5}$ black 
hole. This means that the Hubble equation knows the thermodynamics of the CFT. In this paper we have considered the dynamics of a 4-dimensioanl timelike FRW brane propagating in an 5-dimensional AdS bulk space containing a Schwarschild black hole. Taking into account the semiclassical corrections to the black hole entropy that arise as a result of the self-gravitational effect, and employing the AdS/CFT correspondence, we obtained the self-gravitational corrections to the Cardy-Verlinde formula. A welcomed but not unexpected result was that the modified entropy doesn't violate any entropy bound since the additional term due to the self-gravitation effect is subtractive. These selfgravitational corrections to the Cardy-Verlinde entropy formula express the existence of a deep connection between semi-classical thermodynamics and Anti de Sitter holography. Furthermore, the self-gravitational corrections to the associated Friedmann-like brane equations are obtained. The additional term in the Hubble equation due to the selfgravitation effect goes as $a^{-6}$. Thus, the self-gravitational corrections act as a source of stiff matter contrary to standard FRW cosmology where the charge of the black hole plays this role. A very interesting feature of the solutions of FRW equation is that they all (for $k=0, \pm 1$ ) have a non-vanishing minimum value for the scale factor. That is to say, as long as the self-gravitational correction is non-vanishing, a singularity will certainly be avoided. As a consequence, we found the cosmological evolution of the universe to be regular, with a smooth transition between a contracting and an eventual expanding phase. We presented exact cosmological solutions for a (open, flat, and closed) critical brane.

\section{References}

[1] R.K. Kaul and P. Majumdar, Phys. Rev. Lett. 84, 5255 (2000).

[2] S. Carlip, Class. Quant. Grav. 17, 4175 (2000).

[3] S. Das, P. Majumdar and R.K. Bhaduri, Class. Quant. Grav. 19, 2355 (2002).

[4] S. Das, R.K. Kaul and P. Majumdar, Phys. Rev. D63, 044019 (2001).

[5] A.J.M. Medved and G. Kunstatter, Phys. Rev. D63, 104005 (2001).

[6] D. Birmingham, I. Sachs and S. Sen, Int. J. Mod. Phys. D10, 833 (2001).

[7] T.R. Govindarajan, R.K. Kaul and V. Suneeta, Class. Quant. Grav. 18, 2877 (2001).

[8] M. Cavaglia and A. Fabbri, Phys. Rev. D65, 044012 (2002).

[9] A.J.M. Medved, Class. Quant. Grav. 19, 2503 (2002).

[10] S. Mukherji and S. S. Pal, JHEP 0205, 026 (2002).

[11] S. Das, hep-th/0207072.

[12] G. Gour, Phys. Rev. D66, 104022 (2002).

[13] A. Chatterjee and P. Majumdar, gr-qc/0303030.

[14] J. E. Lidsey, S. Nojiri, S. D. Odintsov and S. Ogushi, Phys. Lett. B544, 337, (2002). 
[15] G. Gour, A. J. M. Medved, gr-qc/0305018.

[16] S. Nojiri, S. D. Odintsov, S. Ogushi, hep-th/0212047.

[17] M. R. Setare, Phys. Lett. B573, 173 (2003).

[18] M. R. Setare, Eur. Phys. J. C33, 555, (2004).

[19] S. Carlip, Class. Quant. Grav. 15, 3609, (1998).

[20] S.W. Hawking, Commun. Math. Phys. 43 (1975) 199.

[21] P. Kraus and F. Wilczek, Nucl. Phys. B 433 (1995) 403, gr-qc/9408003 P. Kraus and F. Wilczek, Nucl. Phys. B 437 (1995) 231, hep-th/9411219 E. Keski-Vakkuri and P. Kraus, Nucl. Phys. B 491 (1997) 249, hep-th/9610045; M.K. Parikh and F. Wilczek, Phys. Rev. Lett. 85 (2000) 5042, hep-th/9907001.

[22] Y. Kwon, Il Nuovo Cimento B 115 (2000) 469; S. Hemming and E. KeskiVakkuri, Phys. Rev. D 64 (2001) 044006, gr-qc/0005115, E.C. Vagenas, Phys. Lett. B 503 (2001) 399, hep-th/0012134 E.C. Vagenas, Phys. Lett. B 533 (2002) 302, hep-th/0109108; A.J. Medved, Class. Quant. Grav. 19 (2002) 589, hep-th/0110289; E.C. Vagenas, Phys. Lett. B 559 (2003) 65, hep-th/0209185; M.K. Parikh, hep-th/0402166; M. R. Setare and E. C. Vagenas, hep-th/0405186.

[23] D. Amati, M. Ciafaloni and G. Veneziano, Phys. Lett. B216, 41 (1989); D. Amati, M. Ciafaloni and G. Veneziano, Nucl. Phys. B347, 550 (1990), K. Konishi, G. Paffuti and P. Provero, Phys. Lett. B234, 276 (1990).

[24] M. Maggiore, Phys. Rev. D49, 5182 (1994); M. Maggiore, Phys. Lett. B319, 83 (1993).

[25] N. Arkani-Hamed, S. Dimopoulos, G. Dvali, Phys. Lett. B429, 263, (1998).

[26] L. Randall, R. Sundrum, Phys. Rev. Lett. 83, 3370, (1999).

[27] L. Randall, R. Sundrum, Phys. Rev. Lett. 83, 4690, (1999).

[28] J. M. Maldacena, Adv. Theor. Math. Phys. 2, 231, (1998).

[29] E. Witten, Adv. Theor. Math. Phys. 2, 253, (1998).

[30] S. S. Gubser, I. R. Klebanov, A. M. Polyakov, Phys. Lett. B428, 105, (1998).

[31] A. Strominger, JHEP 0110 (2001) 034, hep-th/0106113 A. Strominger, JHEP 0111 (2001) 049, hep-th/0110087; For review, see M. Spradlin, A. Strominger and A. Volovich, hep-th/0110007; S. Nojiri and S.D. Odintsov, Phys. Lett.B 519 (2001) 145, hep-th/0106191; S. Nojiri and S.D. Odintsov, JHEP 0112 (2001) 033, hep-th/0107134; S. Nojiri, S.D. Odintsov and S. Ogushi, Phys. Rev. D 65 (2002) 023521, hep-th/0108172; S. Nojiri and S.D. Odintsov, Phys. Lett. B 523 (2001) 165, hep-th/0110064; D. Klemm, Nucl. Phys. B 625 (2002) 295, hep-th/0106247; T. Shiromizu, D. Ida and T. Torii, JHEP 0111 (2001) 010, hep-th/0109057; C.M. Hull, JHEP 0111 (2001) 012, hep-th/0109213 B. McInnes, Nucl. Phys. B 627 (2002) 311, 
hep-th/0110062 Y. S. Myung, Mod. Phys. Lett. A 16 (2001) 2353, hep-th/0110123; E. Halyo, JHEP 0203 (2002) 009, hep-th/0112093 C. P. Burgess, F. Quevedo, R. Rabadan, G. Tasinato and I. Zavala, JCAP, 0402, 008, (2004); O. Obregn, L. Patio and H. Quevedo. Phys. Rev. D68, 026002, (2003); M. R. Setare, Mod. Phys. Lett. A 17 (2002) 2089, hep-th/0210187; M. R. Setare and M. B. Altaie, Eur. Phys. J. C 30 (2003) 273, hep-th/0304072 M. R. Setare, hep-th/0405010.

[32] S. Nojiri, S.D. Odintsov and S. Ogushi, Int. J. Mod. Phys. A 17 (2002) 4809, hep-th/0205187.

[33] A. Hebecker, J. March-Russell, Nucl. Phys. B608, 375, (2001).

[34] I. Savonije and E. Verlinde, Phys. Lett. B 507 (2001) 305, hep-th/0102042.

[35] A. Padilla, Phys. Lett. B528, 274, (2002).

[36] A. Padilla, hep-th/0210217.

[37] M. R. Setare and R. Mansouri, Int. J. Mod. Phys. A 18 (2003) 4443, hep-th/0210252.

[38] E. Witten, Adv. Theor. Math. Phys. 2, 505, (1998).

[39] E. Verlinde, hep-th/0008140.

[40] M. R. Setare and E. C. Vagenas, Phys.Rev.D 68 (2003) 064014, hep-th/0304060.

[41] M. R. Setare and E. C. Vagenas Phys. Lett. B584 (2004) 127, hep-th/0309092.

[42] V. Balasubramanian, J. de Boer and D. Minic, Phys. Rev. D 65 (2002) 123508, hep-th/0110108.

[43] A.J.M. Medved, Phys. Rev. D 66 (2002) 124009, hep-th/0207247.

[44] M. Cvetič, S. Nojiri and S.D. Odintsov, Nucl. Phys. B 628 (2002) 295, hep-th/0112045; S. Nojiri and S.D. Odintsov, gr-qc/0112066.

[45] S. Mignemi, to appear in Phys. Rev. D, hep-th/0307205.

[46] S. Mukherji and M. Peloso, Phys. Lett. B 547 (2002) 297, hep-th/0205180

[47] A.J.M. Medved, JHEP, O305, 008, (2003), hep-th/0301010.

[48] A.J.M. Medved, hep-th/0205251; Y. Shtanov and V. Sahni, Phys. Lett. B 557 (2003) 1, gr-qc/0208047.

[49] A.K. Biswas and S. Mukherji, JHEP 0103 (2001) 046, hep-th/0102138.

[50] A.J.M. Medved, hep-th/0111182. 